\title{
A IMPORTÂNCIA DE UM ESTUDO DINÂMICO PARA A SIMULAÇÃO DO ESCOAMENTO DO AÇO LÍQUIDO NO DISTRIBUIDOR DE LINGOTAMENTO CONTÍNUO*
}

\author{
Felipe das Dores Machado ${ }^{1}$ \\ Magda Beatriz Galant François ${ }^{2}$ \\ Antônio Cezar Faria Vilela
}

\begin{abstract}
Resumo
O estudo de simulação do escoamento do aço líquido no distribuidor requer não somente um grande conhecimento técnico dos critérios de similaridade, mas também uma correta aplicação das ferramentas disponíveis de simulação dos fenômenos físicos envolvidos. A complexidade do escoamento, tendo em vista a interação das inúmeras variáveis envolvidas, torna necessário um estudo dinâmico que utilize diferentes métodos que abranjam a visão de um todo do escoamento, aumentando as oportunidades de uma conclusão mais assertiva sobre os fenômenos ocorridos nas reais condições encontradas na indústria. Este trabalho tem o objetivo apresentar uma nova abordagem sobre a simulação da remoção de inclusões do escoamento do aço líquido em modelos físicos do distribuidor de lingotamento contínuo e como resultado será mostrado um estudo de caso comprovando que a utilização de um único método de modelagem física pode levar a compreensões equivocadas do escoamento.
\end{abstract}

Palavras-chave: Modelagem física; Escoamento; Distribuidor.

THE IMPORTANCE OF A DINAMIC STUDY TO THE SIMULATION OF MELT FLOW IN CONTINUOUS CASTING TUNDISH

\section{Abstract}

The simulation study of the melt flow in the tundish requires not only a great technical knowledge of similarity criteria but also a correct application of the tools available to simulate the physical phenomena involved. The complexity of the melt flow, in view of the interaction of many variables involved, turns necessary a dynamic study using different methods covering the vision of a whole flow. This approach increases the opportunities for a more assertive conclusion about the phenomena under actual conditions found in the industry. This work aims to show a new approach to the simulation of the inclusion removal in physical models of the continuous casting tundishes. It will show a case study demonstrating that the use of a single method of physical model can lead to erroneous flow understandings.

Keywords: Physical Model; Melt flow; Tundish.

\footnotetext{
Msc. Eng., Doutorando; LaSid; Departamento de Metalurgia; PPGE3M, UFRGS, Canoas, RS, Brasil.

Prof. Dr. INPL, Pesquisadora LaSid, UFRGS, Prof. ULBRA, Porto Alegre, RS, Brasil

Prof. Dr.-Ing., LaSid, Departamento de Metalurgia, PPGE3M, UFRGS, Porto Alegre, RS, Brasil.
} 


\section{INTRODUÇÃO}

O uso de modelos físicos e/ou numéricos têm sido de grande valor para os estudos de escoamento [1-3]. A realização desses estudos diretamente na planta, é de difícil implementação, em grande parte devido a opacidade do distribuidor e do aço que impossibilitam a visualização do escoamento e também em função dos riscos de segurança pois as temperaturas de trabalho são geralmente entre 1550 e $1600^{\circ} \mathrm{C}$. Desta forma, utilizar água como fluido simulador de aço líquido facilita o estudo, tendo em vista que suas propriedades físicas e químicas permitem a visualização do escoamento e um comportamento fluidodinâmico similar à do aço líquido [4].

Para que os resultados, tanto dos modelos físicos quanto numéricos, possam ser utilizados com alguma segurança, eles devem obedecer a critérios de similaridade (geométrica, dinâmica, cinemática, térmica e química) e as respectivas forças que governam os fenômenos envolvidos [5-9]. Pode-se inferir que, devido à diversidade dos fenômenos, é impossível que se possa manter, em um mesmo modelo físico, todos os critérios de similaridade e obter resultados confiáveis para diferentes fenômenos com o mesmo rigor [5-8,10,11].

A modelagem física traz como ferramentas de estudo diferentes metodologias e abordagens que irão fornecer resultados diferentes para cada fenômeno que se deseja simular. Alguns podem ser listados: distribuição de tempos de residência na determinação dos tempos e volumes característicos, visualização de linhas de fluxo na identificação das zonas mortas, curto-circuito e caminhos preferenciais, emulsificação de escória para visualização do arraste de escória para o interior do escoamento e remoção de inclusões para a quantificação das inclusões que não flotam no distribuidor e, consequentemente, seguem em direção ao molde.

Diante desses métodos, o diferencial em um bom estudo fluidodinâmico do aço líquido é possuir um estudo dinâmico capaz de abranger o máximo dos fenômenos envolvidos no escoamento de forma a criar uma metodologia da combinação dos ensaios anteriormente citados que permitam o correto entendimento do escoamento como um todo. É claro que se deve lembrar das limitações de cada ensaio e da escolha correta das relações entre os respectivos critérios de similaridade e o papel das forças que interagem em cada tipo de fenômeno.

\section{MATERIAIS E MÉTODOS}

Para este trabalho serão aplicados três ensaios de modelagem física em um modelo isotérmico e construído em acrílico de distribuidor, pertencente ao Laboratório de Siderurgia da UFRGS, são eles: visualização de linhas de fluxo, ensaio de distribuição de tempos de residência e o ensaio de remoção de inclusões. Para comparações, o distribuidor é montado em quatro configurações. São elas: sem tubo longo e sem barreiras, sem tubo longo e com barreiras, com tubo longo e sem barreiras e com tubo longo e com barreiras. A representação esquemática do modelo físico e das configurações podem ser vistos na Figura 1. 


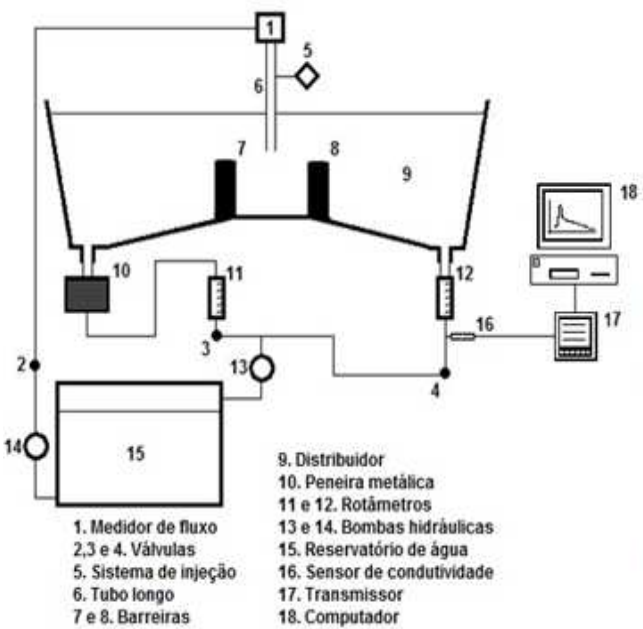

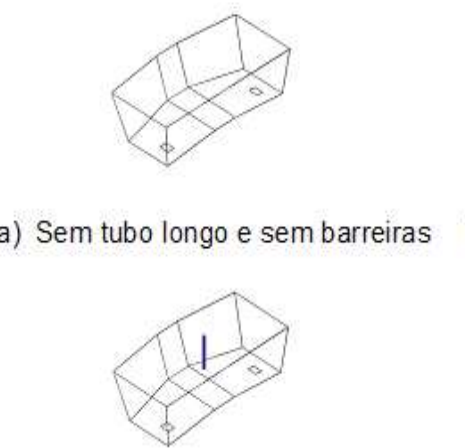

c) Com tubo longo e sem barreiras b) Sem tubo longo e com barreiras

Figura 1. Ilustrações em três dimensões do distribuidor com as configurações estudadas nos ensaios de modelagem física.

Os dois primeiros ensaios, visto que os parâmetros analisados remetem à remoção de inclusões, dão suporte, de forma dinâmica, ao entendimento dos resultados do ensaio de quantificação de remoção de inclusões. A metodologia de cada ensaio será apresentada a seguir. Entretanto, para melhores informações, recomenda-se a leitura da dissertação de Felipe D. Machado [9].

A motivação do ensaio de linha de fluxo é estudar qualitativamente os escoamentos, identificando as características do escoamento que são favoráveis e desfavoráveis (ex. curto-circuito, volume morto, etc.) para a remoção de inclusões. $O$ ensaio é realizado com o distribuidor em regime permanente e com velocidade de lingotamento de $2,2 \mathrm{~m} / \mathrm{min}$. Para a visualização do escoamento é realizada uma injeção (tipo pulso de 3 segundos) de aproximadamente $400 \mathrm{ml}$ de volume de uma solução de flutuação neutra de permanganato de potássio $(\mathrm{KMnO} 4)$ após a estabilização do escoamento (10 minutos). O caminho em que o corante escoa no distribuidor é acompanhado por meio de câmeras filmadoras posicionadas nas três vistas do modelo.

O ensaio de distribuição de tempos de residência tem como motivação o estudo quantitativo do escoamento, determinando parâmetros, como tempo mínimo de residência, tempo médio de residência e volume característicos do escamento (volume pistonado, mistura, etc.) que estão diretamente relacionados à remoção de inclusões. O método empregado consiste na injeção de um traçador ácido e leitura da condutividade na saída do modelo ao longo do tempo de ensaio (30 minutos). As condições de injeção são as mesmas descritas para o ensaio de linhas de fluxo.

O ensaio de remoção de inclusões consiste na injeção de $30 \mathrm{~g}$ de partículas de polietileno em solução de álcool etílico absoluto para a simulação de inclusões de alumina nas mesmas condições de injeção do ensaio de linhas de fluxo. Para quantificar as inclusões passantes pelos veios, uma peneira metálica é instalada na saída dos veios e que ao fim do ensaio (dois tempos de residência teórica) é retirada, secada e pesada para o calculado do percentual de remoção de inclusões.

\section{RESULTADOS E DISCUSSÃO}

A Tabela 1 mostra um resumo dos principais resultados obtidos para os ensaios de linhas de fluxo, distribuição de tempos de residência e remoção de inclusões. A seguir são discutidas as configurações em ordem de melhor para pior configuração. 
Dentro das limitações da modelagem física e sob o ponto de vista da remoção de inclusões, a melhor configuração é a sem barreiras e sem tubo longo, apresentado $2,56 \%$ de inclusões passantes. Essa configuração apresenta escoamento preferencialmente ascendente em direção à superfície e arraste de bolhas para o interior do escoamento favorecendo a remoção de inclusões. Apesar de possuir curto-circuito de $3,4 \%, 3^{\circ}$ melhor adimensional de tempo médio de residência $(\bar{\theta}=0,520), 3^{\circ}$ melhor tempo mínimo de residência $\left(t_{\min }=18,20 \mathrm{~s}\right)$ e $3^{\circ}$ melhor relação de $\mathrm{Vpd} / \mathrm{Vd}$ igual a 0,187 , a escolha dessa configuração atribui-se principalmente a existência das bolhas que podem ter levado ao menor resultado de inclusões passantes pelo veio.

De acordo com os ensaios realizados neste trabalho, a qualidade do aço produzido utilizando as barreiras tenderia a piorar na configuração sem tubo longo. Visto que o volume de curto-circuito aumenta de 3,4 para 9,1\%, o adimensional de tempo médio diminui de 0,520 para 0,487 , o tempo mínimo passa de 18,20 para $18,21 \mathrm{~s}$, a relação $\mathrm{Vpd} / \mathrm{Vd}$ cai de 0,187 para 0,183 e o porcentual de inclusões passantes aumenta de 2,08 para $2,51 \%$. Mesmo assim, a diferença para o $3^{\circ}$ melhor resultado de remoção de inclusões é bastante grande $(21,05 \%)$, atribui-se principalmente a existência das bolhas. Isso não significa que outras barreiras empregadas nesse distribuidor não seriam favoráveis à remoção de inclusões, como barreiras mais altas, em posições diferentes e/ou com furos. Isso significa que a posição e o formato das barreiras não apresentaram bons resultados para a remoção de inclusões.

A configuração com tubo longo e com barreiras apresenta um escoamento preferencialmente ascendente, sem a presença de curto-circuito, com o maior tempo mínimo ( $\left.t_{\min }=44,29 \mathrm{~s}\right)$ e médio de residência $(\bar{\theta}=0,590)$ e melhor relação $\mathrm{Vpd} / \mathrm{Vd}$ dentre as configurações estudadas $(\mathrm{Vpd} / \mathrm{Vd}=0,293)$. Mesmo apresentando os melhores resultados no ensaio de linhas de fluxo e distribuição de tempo de residência, os resultados de remoção de inclusões ficaram em $3^{\circ}$ lugar com $21,05 \%$. A configuração com tubo longo e sem barreiras se mostrou como a pior configuração sob o ponto de vista da remoção de inclusões, apresentado $29,76 \%$ de inclusões passantes. A configuração apresentou escoamento preferencialmente descendente, pior resultado de curto-circuito $(11,1 \%)$, pior resultado de tempo mínimo de residência $\left(t_{\min }=9,69 \mathrm{~s}\right)$. Apesar de apresentar $2^{\circ}$ melhor resultado de adimensional de tempo médio $(\bar{\theta}=0,550)$ e $2^{\circ}$ melhor resultado de relação $\mathrm{Vpd} / \mathrm{Vd}$ igual a 0,250 . Novamente comparados às configurações sem tubo longo, a falta de bolhas no interior do escoamento faz com que as inclusões passantes aumentem. 
Tabela 1. Resumo dos resultados obtidos nos ensaios de linhas de fluxo, distribuição de tempos de residência e remoção de inclusões.

\begin{tabular}{|c|c|c|c|c|}
\hline & $\begin{array}{c}\text { Sem barreiras e sem tubo } \\
\text { longo }\end{array}$ & $\begin{array}{c}\text { Com barreiras e sem tubo } \\
\text { longo }\end{array}$ & $\begin{array}{c}\text { Sem barreiras e com tubo } \\
\text { longo }\end{array}$ & $\begin{array}{c}\text { Com barreiras e com tubo } \\
\text { longo }\end{array}$ \\
\hline \multirow{2}{*}{$\begin{array}{l}\text { Fluxo ascendente em direção } \\
\text { à superfície } \\
\text { (Tempo em segundos após a } \\
\text { injeção do corante) }\end{array}$} & SIM & SIM & NÃO & SIM \\
\hline & $4 \mathrm{~s}$ & $4 s$ & $4 \mathrm{~s}$ & $16 \mathrm{~s}$ \\
\hline Arraste de bolhas & SIM & SIM & NÃO & NÃO \\
\hline $\begin{array}{l}\text { Presença de volume de curto- } \\
\text { circuito }\end{array}$ & SIM $-3,4 \%$ & SIM $-9,1 \%$ & SIM $-11,1 \%$ (Pior resultado) & NÃO - $0 \%$ (Melhor resultado) \\
\hline $\begin{array}{l}\text { Adimensional do tempo } \\
\text { médio de residência (valor } \\
\text { médio entre as velocidades) }\end{array}$ & 0,520 & 0,487 (Pior resultado) & 0,550 & 0,590 (Melhor resultado) \\
\hline $\begin{array}{l}\text { Tempo mínimo de residência, } \\
\text { em segundos, (valor médio } \\
\text { entre as velocidades) }\end{array}$ & 18,20 & 18,21 & 9,69 (Pior resultado) & 44,29 (Melhor resultado) \\
\hline $\begin{array}{l}\text { Relação Vpd/Vd (valor médio } \\
\text { entre as velocidades) }\end{array}$ & 0,187 & 0,183 (Pior resultado) & 0,250 & 0,293 (Melhor resultado) \\
\hline$\%$ inclusões passantes & 2,56\% (Melhor resultado) & $3,01 \%$ & $29,76 \%$ (Pior resultado) & $24,29 \%$ \\
\hline
\end{tabular}

Observação: Na tabela são indicados e destacados os melhores (verde) e os piores resultados (vermelho).

\subsection{A Importância do Estudo Dinâmico na Aplicação Apresentada neste Trabalho}

Como apresentado anteriormente, a melhor configuração encontrada é a sem tubo longo e sem barreiras. No entanto, se fosse realizado somente o ensaio de linhas de fluxo, a melhor configuração seria a com tubo longo e com barreiras. Mesmo aplicando o ensaio de distribuição de tempos de residência, a mesma conclusão seria encontrada. Isso ocorre por que a aplicação exclusiva dos ensaios de linhas de fluxo e/ou distribuição de tempos de residência não simulam o fenômeno de captura de inclusões não metálicas por presença de bolhas no escoamento (Figura 2). Este fenômeno é muito discutido na literatura $[3,12,13]$ e é fundamental simulá-lo quando presente nos escoamentos estudados.

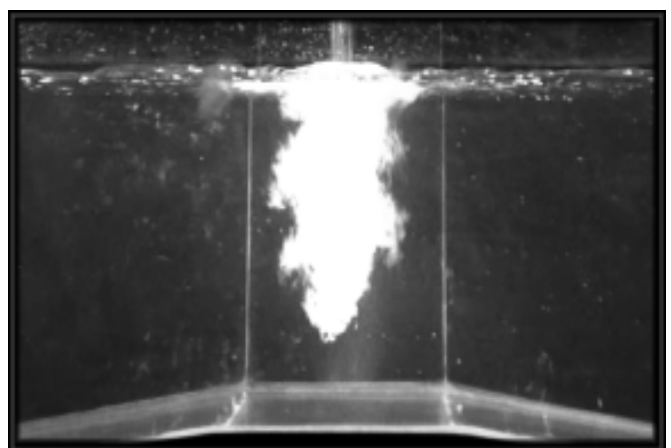

Figura 2. Visualização em modelo físico do arraste de bolhas para o seio do escoamento gerado pela utilização de jato aberto (sem tubo longo).

Em uma outra análise, quando se comparam as configurações sem tubo longo alternando a presença ou não de barreiras, é possível afirmar que não se pode comparar qual das configurações apresentam melhores condições para remoção de inclusões. Somente quando se utiliza o ensaio de distribuição de tempos de residência que se pode afirmar que o curto circuito é maior na configuração com 
barreiras. Mais importante ainda é que quando se apresentam os resultados de remoção de inclusões, essa condição praticamente não representa muita diferença na remoção de inclusões. Novamente, pelo mesmo motivo presentado no parágrafo anterior.

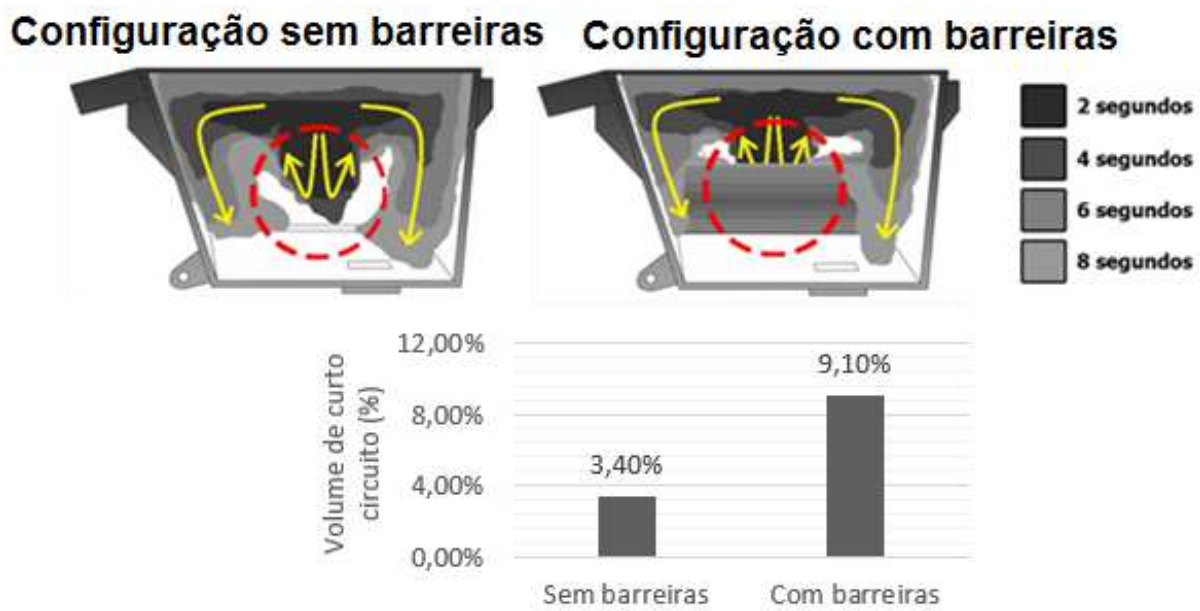

Figura 3. Resultados de linhas de fluxo e distribuição de tempos de residência nas configurações sem tubo longo e com/sem barreiras.

Essas duas breves análises mostraram que facilmente, utilizando as ferramentas de modelagem física, pode-se obter conclusões precipitadas quanto a remoção de inclusões nos distribuidores de lingotamento contínuo. Na prática, a escolha errada de uma configuração interna do distribuidor leva a um escoamento que pode ser prejudicial e, consequentemente, uma piora na limpeza inclusionária no aço poderá ocorrer. Esses erros podem trazer uma certa desconfiança dos pesquisadores e da indústria para uma técnica que a primeira vista, para os que não a conhecem, é bastante diferente da realidade.

\section{CONCLUSÕES}

Conclui-se que é necessária a utilização de um estudo dinâmico que envolva diferentes técnicas de modelagem física e que abranjam o máximo dos fenômenos envolvidos. Isso faz com que se evite equívocos quanto a caracterização de escoamentos internos de distribuidor sob o ponto de vista da remoção de inclusões.

Quanto às configurações estudadas, conclui-se que, ao comparar a configuração sem tubo longo e sem barreiras com a configuração sem tubo longo e com barreiras, ocorre:

$>$ Permanência do curto-circuito e aumento do mesmo de 3,4 para 9,1\%;

$>$ Diminuição do adimensional de tempo médio de residência de 0,520 para 0,487

$>$ Diminuição na relação $\mathrm{Vpd} / \mathrm{Vd}$ de 0,187 para 0,183 ;

> Aumento do porcentual de inclusões passantes de 2,56 para 3,01\%.

A partir desses resultados conclui-se que para os casos em que se utiliza o jato aberto e a reoxidação do banho não é limitante na qualidade do aço produzido, não é aconselhável modificar o escoamento utilizando as barreiras deste estudo. Ou seja, mudar da configuração sem tubo longo e sem barreiras para a configuração sem tubo longo com barreiras não é uma boa opção. 
Ao utilizar no distribuidor a configuração com tubo longo e sem barreiras obtêm-se, com os resultados de linhas de fluxo, distribuição de tempos de residência e remoção de inclusões, que:

$>$ Escoamento preferencialmente descendente;

$>$ Pior resultado de fração de volume de curto-circuito igual a 11,1\%;

$>$ Pior resultado de tempo mínimo de residência no valor de 9,69 s;

$>$ Pior resultado de porcentual de inclusões passantes pelo veio elevado e igual a $29,76 \%$.

Portanto, conclui-se que essa configuração é a pior dentre as quatro configurações de distribuidor testadas neste trabalho. No entanto, ao comparar a configuração com tubo longo e sem barreiras com a configuração com tubo longo e com barreiras ocorre:

$>$ Modificação do escoamento de descendente para ascendente em direção à superfície;

$>$ Eliminação do curto-circuito de 11,1 para 0\%;

> Aumento do adimensional de tempo médio de residência de 0,550 para 0,590;

$>$ Aumento do tempo mínimo de residência de 9,69 para 44,29 s;

> Aumento da relação Vpd/Vd de 0,250 para 0,293;

$>$ Diminuição o porcentual de inclusões passantes pelo veio de 29,76 para $24,29 \%$.

Porém, a melhor configuração estudada neste trabalho é a configuração sem tubo longo e sem barreiras que apresentou $2,56 \%$ de inclusões passantes pelo veio. Além de apresentar os seguintes resultados:

$>$ Escoamento preferencialmente ascendente;

$>$ Arraste de bolhas para o interior do escoamento favorecendo a remoção de inclusões;

$3^{\circ}$ melhor adimensional de tempo médio de residência $(\overline{\boldsymbol{\theta}}=0,520)$;

$>3^{\circ}$ melhor relação de $\mathrm{Vpd} / \mathrm{Vd}$ igual a 0,187.

\section{Agradecimentos}

Ao CNPq pela bolsa concedida ao Felipe das Dôres Machado durante o desenvolvimento do mestrado no Programa de Pós Graduação de Engenharia de Minas, Metalúrgica e Materiais (PPGE3M) da UFRGS.

\section{REFERÊNCIAS}

1 Joo, S.; Guthrie, R. I. L. Inclusion behavior and heat-transfer phenomena in steelmaking tundish operations Part I. aqueous. Metallurgical transactions, v. 24, p. 755-65, 1993.

2 Cho, J.; Lee, H. Cold model study on inclusion removal from liquid steel using fine gas bubbles. The iron and steel institute of Japan international, v. 41, p. 151-7, 2001.

3 Rogler, J. P.; Heaslip, L. J.; Mehrvar, M. Physical modelling of inclusion removal in a tundish by gas bubbling. Canadian metallurgical quarterly, v. 44, p. 357-68, 2005.

4 Mazumdar, D.; Guthrie, I. L. R. The physical and mathematical modeling of continuous casting tundish systems. ISIJ Intern., v. 39, n. 6, p. 524-47, 1999.

5 Sahai, Y.; Emi, T. Tundish technology for clean steel production. World Scientific Publishing Company, 2007.

6 Szekely, J.; Evans, J. W.; Brimacombe, J. K. The mathematical and physical modeling of primary metals procesing operations. Wiley-Interscience, Nova York, 1988.

7 Mazumdar, D.; Evans, J. W. Modeling of steelmaking process. CRC Press, 2009. 
8 Szekely, J. Fluid flow phenomena in metals processing. Departament of materials science and engineering, Massachussetts Institute of Technology. Academic Press, 1979.

9 Machado, F. D. Modelagem física de remoção de inclusões em distribuidor de lingotamento contínuo de tarugos. Dissertação de mestrado a ser defendida em 24 de março de 2014. Porto Alegre: Universidade Federal do Rio Grande do Sul, 2014.

10 Sahai, Y.; Emi, T. Criteria for water modeling of melt flow and inclusion removal in continuous casting tundishes. The iron and steel institute of Japan international, v. 36, p. 1166-73, 1996.

11 Sinha, A. K.; Sahai, Y. Mathematical modeling of inclusion transport and removal in continuous casting tundishes. The iron and steel institute of Japan international, v. 33, p. 556-65, 1993.

12 Chattopadhyay, K.; Isac, M.; Guthrie, R. I. L. Physical and Mathematical Modelling to Study the Effect of Ladle Shroud Mis-alignment on Liquid Metal Quality in a Tundish. The iron and steel institute of Japan international, v. 51, p. 759-68, 2011.

13 Zhang, M. J., et al. Physical e mathematical modeling of inclusion removal with gas bottom-blowing in continuos casting tundish. Journal of mining and metallurgy, v. 47, p. 37-44, 2011. 